\title{
Electron Cryo-microscopy and Image Reconstruction of Adeno-Associated Virus Type 2 empty Capsids
}

\author{
B. Böttcher ${ }^{*}$, S. Kronenberg ${ }^{* *}$ and J. Kleinschmidt ${ }^{* *}$ \\ ${ }^{*}$ EMBL, Meyerhofstrasse 1, D-69117 Heidelberg, Germany \\ *** DKFZ, Im Neuenheimerfeld 242, D-69120 Heidelberg, Germany
}

Adeno-associated virus type 2 (AAV-2) is a human parvovirus. The virus capsid is composed of three different structural proteins, VP1, VP2 and VP3 which have molecular masses of $87 \mathrm{kDa}, 72$ $\mathrm{kDa}$ and $62 \mathrm{kDa}$ respectively and differ in their $\mathrm{N}$-terminal amino-acid sequences. The likely molar ratio of VP1, VP2 and VP3 is 1:1:8. In total sixty copies of these structural proteins occupy symmetrically equivalent positions in an icosahedrally arranged protein shell.

We have investigated empty capsids of AAV-2 by electron cryo-microscopy and icosahedral image reconstruction 1 . The three-dimensional map at $10.5 \AA$ resolution showed sets of three elongated spikes surrounding the three-fold symmetry axes and narrow empty channels at the five-fold axes (Figure 1A, 1B). The inside of the capsid superimposed with the previously determined structure of the canine parvovirus ${ }^{2}$, whereas the outer surface showed clear discrepancies. Globular structures at the inner surface of the capsid at the two-fold symmetry axes were identified as possible positions for the N-terminal extensions of VP1 and VP2 (Figure 1B, arrows).

The data was collected on a Philips CM 120 Biotwin at $100 \mathrm{kV}$. This microscope is designed for high contrast at low spatial frequencies by using a lens with a high $C_{s}$ value $\left(C_{s}=6.4 \mathrm{~mm}\right)$. The payoff of this design together with the use of a standard $\mathrm{LaB}_{6}$-filament was a loss in high resolution information, which depended on the defocus and the illumination conditions used for taking a particular micrograph. The dampening of higher spatial frequencies is described by an envelope function $\mathrm{K}_{\mathrm{s}}$ which modulates the contrast-transfer-function ctf (Figure 1C). $\mathrm{K}_{\mathrm{s}}$ is different for each micrograph taken at a different defocus or under different illumination conditions. To allow for these variations of $\mathrm{K}_{\mathrm{s}}, \mathrm{K}_{\mathrm{s}}$ was included in the previously described ctf-correction ${ }^{3}$ of the observed values of a particular frequency component F. For evaluating the altered ctf-correction-algorithm, the Fourier-shell-correlation between two maps calculated from half of the particle images each was determined. Figure 1D shows the Fourier-shell-correlation after correction for the ctf including the calculated $\mathrm{K}_{\mathrm{s}}$ for each micrograph in trace 1 and after correction for the ctf neglecting $\mathrm{K}_{\mathrm{s}}$ (by setting it to one) in trace 2 . The Fourier-shell-correlation values were clearly higher when $\mathrm{K}_{\mathrm{s}}$ was included in the correction-algorithm, highlighting the importance of the $\mathrm{K}_{\mathrm{s}}$-correction.

The overall dampening of the high resolution information, caused by the lack of spatial coherence of the microscope was evident in the fall off of $\sum C t f_{i}^{2} w_{i}$ with increasing spatial frequencies (trace 3). The value of $\sum C t f_{i}^{2} w_{i}$ is a direct measure for expected information transfer of the microscope for the average of the observed data and can range from zero for no transfer up to one for transfer without loss due to the microscope settings. A considerable loss in the transfer of information was already apparent at frequencies of $1 / 25 \AA^{-1}$. However, this drop was not reflected in the fall-off of the Fourier-shell-correlation (trace 1), illustrating that information can be recovered even though it is only weakly transferred.

\section{References:}

[1] Kronenberg, S., Kleinschmidt, J.A. and Böttcher, B. (2001) EMBO Rep 2, 997-1002. 
[2] Xie, Q. and Chapman, M.S. (1996) J Mol Biol 264, 497-520.

[3] Böttcher, B. and Crowther, R.A. (1996) Structure 4, 387-394.

[4] Reimer, L. (1993) Transmission Electron Microscopy. Berlin, Heidelberg, Springer Verlag.

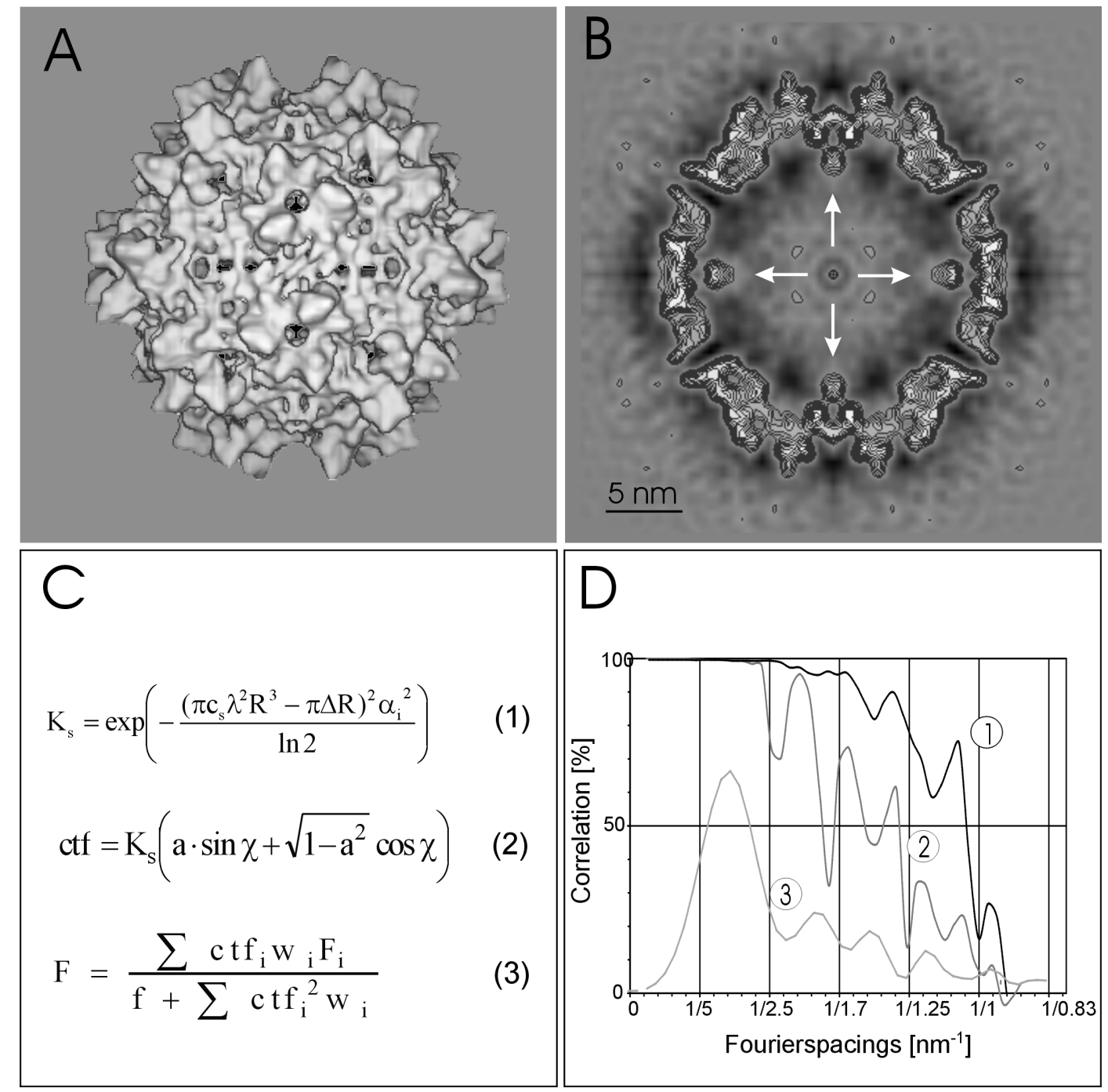

Figure 1: A) Surface representation of the three-dimensional map of empty AAV-2 capsids at 10.5 $\AA$ resolution. B) Equatorial slice of the map. C) 1) Envelope function $K_{s}$ caused by the spatial coherence of the electron source ${ }^{4}\left(C_{s}\right.$ spherical aberration coefficient, $\lambda$ wavelength of electrons, $R$ spatial frequency, $\Delta$ defocus, $\alpha$ illumination half angle). 2) contrast transfer function $c t f$ modulated by the envelope function $K_{s}(\chi$ phase-shift; $a$ fractional amplitude contrast). 3) Combination of the observed Frequency components $F_{i}$ in $i$ reconstructions of particle images from $i$ different micrographs to a $c t f$ corrected Frequency component $F$ ( $w$ fractional weight of each map, $f$ factor akin to signal to noise ratio in a Wiener filter) ${ }^{3}$. D) Fourier-Shell-Correlation of the ctf-corrected data using the calculated value for $K_{s}$ (trace 1) and with $K_{s}$ set to 1 (trace 2). Trace 3 shows $\sum C t f_{i}^{2} w_{i}$, a direct measure for the expected information transfer of the microscope for the average of the observed data. 